\title{
PISA - Mathematische Schülerleistungen im OECD-Vergleich Anzeichen für eine neue Schieflage?
}

\section{von Günter Törner}

Die Diskussion um den schulischen Mathematikunterricht ließ sich in den letzten beiden Jahren zumeist an einer Abkürzung festmachen: TIMSS. Diese dritte internationale Studie über mathematischen und naturwissenschaftichen Unterricht, über die wir mehrfach berichtet haben, brachte zutage, dass die mathematischen Schülerleistungen in Deutschland im internationalen Vergleich im Mittelfeld anzusiedeln sind. Dieser allgemein als unbefriedigend eingestufte Befund - deutscher Mathematikunterricht ist hinsichtlich der Leistungskompetenz der Schüler im Wesentlichen mit dem Unterricht in den Vereinigten Staaten vergleichbar - hat manche Initiativen ausgelöst, viele Diskussionen kontrovers beherrscht und fachdidaktische wie auch padägogische Analysen initiiert, die zu einer selbstkritischen wie fruchtbaren Bestandsaufnahme geführt haben ${ }^{1}$.

Auch das Präsidium der Deutschen-MathematikerVereinigung (DMV) hat sich in diese Diskussion eingebracht. Es verging keine Präsidiumssitzung, auf der nicht das Thema Schule und Lehramt Gegenstand von Diskussionen war. So wurden im letzten Jahr in Abstimmung mit der Bund-LänderKommission (BLK) in allen Bundesländern Ansprechpartner 'Schule und Lehramt' installiert. Mit diesen Aktivitäten will die DMV nachhaltig unterstreichen, dass Verbesserungen des schulischen Mathematikunterrichts, will man ihnen Nachhaltigkeit zubilligen, auch mit Kooperationen mit und Veränderungen auf der universitären Seite einhergehen müssen. Dieser Zusammenhang, zumindest was die fachmathematischen Aspekte und die fachmathematische Ausbildung zukünftiger Lehrer anbetrifft, ist bislang in der bildungspolitischen Diskussion nur unzureichend ins Blickfeld genommen worden.

Nun müssen wir uns nach TIMSS in den nächsten Jahren mit einem neuen Akronym vertraut machen, nämlich mit PISA. Nomen est Omen? Soll da nebenbei an die Stadt erinnert werden, in der die Schieflage eines Turmes immer wieder viele Touristen in den Bann zieht? Dem Vernehmen nach soll aber mittlerweile eine Lösung für den instabilen Turm gefunden worden sein. Fairerweise muss man sagen, dass PISA für das Programme for International Student Assessment steht und Teil eines Indikatorenprogramms INES (Indicators of Educational Systems) ist, das von der Organisation für wirtschaftliche $\mathrm{Zu}$ sammenarbeit und Entwicklung (OECD) verantwortet wird ${ }^{2}$. Die Bundesrepublik Deutschland beteiligt sich an diesem Programm gemäß einer Vereinbarung zwischen dem Bundesministerium für Bildung und Forschung und der Ständigen Kultusministerkonferenz der Länder. Dabei soll PISA alle drei Jahre in rund 30 wichtigen Industriestaaten die Leistungen von 15jährigen Schülerinnen und Schülern in den Bereichen Leseverständnis, Mathematik und Naturwissenschaft messen. Es ist Anliegen von PISA, Kompetenzen zu erfassen, die für eine erfolgreiche Lebensführung notwendig sind. Hierzu gehört unsererseits die Vertrautheit im Umgang mit mathematischen Symbolen und Modellen, weniger das Abtesten von einzelnen Fakten. Im Unterschied zu TIMSS machen in diesen Tests Multiple-Choice-Aufgaben nur noch die Hälfte der Aufgaben aus.

Den internationalen Vorgaben entsprechend, werden in Deutschland in etwa 170 Schulen jeweils 35 15jährige Schülerinnen und Schüler getestet. Zusätzlich zu der internationalen Erhebung werden in Deutschland an einem zweiten Testtag ergänzende nationale Untersuchungen durchgeführt. In diesem ergänzenden Test sollen in den Bereichen Mathematik und Naturwissenschaft lehrplangültige Aufgaben eingesetzt werden, insbesondere solche Problemstellungen, die mit den internationalen Tests nur unzureichend abgedeckt werden.

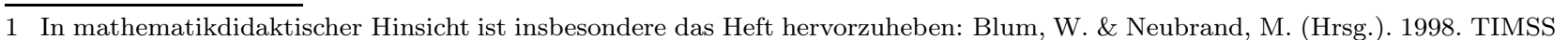
und der Mathematikunterricht. Hannover: Schroedel.

2 vgl. die Publikation: OECD. 1999. Measuring Student Knowledge and Skills - A New Framework for Assessment. Paris: OECD Programme for international student assessment. 
Damit wird deutlich, dass PISA von einem eher ganzheitlichen Verständnis von Mathematik ausgeht. Bereits TIMSS hat hier den Begriff der mathematischen Literalität (mathematics literacy) geboren. Wörtlich lautet im internationalen PISA-Framework die entsprechende Definition für mathematische Kompetenz: Mathematics literacy is an individual's capacity to identify and understand the role that mathematics plays in the world, to make well-founded mathematical judgements and to engage in mathematics, in ways that meet the needs of that individual's current and future life as a constructive, concerned and reflective citizen.

Zugegebenermaßen handelt es sich um eine nicht gerade anspruchslose Zielsetzung, die Wissen gezielt daraufhin untersucht, ob es funktional ist und mit Einsicht und flexibel zur Bearbeitung kontextbezogener Probleme eingesetzt werden kann. Es sei dem Leser überlassen, die angestrebte Mathematics Literacy für Diplomkandidaten zu präzisieren.

Ohne auf Details einzugehen ${ }^{3}$, sei erwähnt, dass für den OECD/PISA-Test vier Aspekte maßgeblich sind, nämlich die beiden Hauptaspekte mathematical competencies und big ideas. Die Aspekte mathematical curricular strands and situations and contexts werden den oben genannten Schwerpunkten nachrangig untergeordnet.

Unter mathematischen Kompetenzen werden mathematische Kenntnisse, Fertigkeiten und Fähigkeiten verstanden, etwa mathematisches Denkvermögen im Allgemeinen, mathematisches Argumentieren, Modellierungsfähigkeiten, Problemlöse- und Problemformulierungsfähigkeiten, Darstellungsfähigkeiten, technische Fertigkeiten und Fähigkeiten zum Gebrauch der mathematischen Sprache, Kommunikationsfähigkeiten über mathematische Inhalte und die Fähigkeit, Hilfsmittel passend einsetzen zu können.

Bedeutsam ist für PISA die Fokusierung auf sogenannte 'big ideas'; Beispiele für 'big ideas' sind etwa chance, change and growth, dependency and relationships, space and shape usw. Für den ersten $\mathrm{Zy}-$ klus von PISA, bei dem Mathematik eine untergeordnete Rolle spielt, werden zwei 'big ideas' herausgegriffen, nämlich change and growth und shape and space. Dieser Ansatz lässt deutlich die Handschrift des Freudenthal-Instituts aus Utrecht erahnen, das wesentlich das Erhebungskonzept gestaltet hat. So überzeugend dieses Konzept auf den ersten Blick ist, zentrale mathematikaffine Aspekte als Leitlinien einer Strukturierung von Unterricht heranzuziehen, so zurückhaltend dürfte die erwünschte Reproduktion durch die schulischen Tests einzustufen sein. Diese 'big ideas' sind meist nicht explizit in den Curricula verankert, obgleich sie zentrale Themenkreise der Mathematik betreffen. Sie prüfen daher schulische Kenntnisse indirekt ab, indem sie allerdings ein vertieftes Verständnis mathematischer Begriffe voraussetzen. Ihr Vorteil, Mathematik nicht themen- oder stoffspezifisch zu durchdringen, sondern fachgebietsübergreifend zu realisieren, dürfte zugleich als Nachteil für die bundesdeutsche Testlandschaft anzusehen sein.

Positiv betrachtet liegt hierin ein Keim, über die Strukturierung des mathematischen Schulstoffs grundlegend nachzudenken, so sehr kann nicht übersehen werden, dass die klassischen Stoffgebietsstrukturen auch hilfreiche Orientierungsfunktionen aufweisen können. Letztlich ist aber das Portionieren in wohlüberschaubare Gebiete nur bedingt weiterentwicklungsfähig, so gerne Lehrer daran festhalten wollen. Neue mathematische Gebiete, die auch auf die Schule abstrahlen, beispielsweise Diskrete Mathematik oder Optimierung, haben es schwer, innerhalb der wohlabgesteckten 'Claims' Platz zu finden. Es kann nicht ignoriert werden, dass hinter dieser stoffgebietsorientierten Aufarbeitung von Mathematik nicht selten unreflektiert didaktische Lehrprinzipien stehen, deren Angemessenheit und Zeitgemäßheit aufgrund allgemeiner neuer lernpsychologischer Einsichten erst zu beweisen wäre.

Was gerade mit Blick auf den schulischen Mathematikunterricht dargelegt wurde, muss auch auf der Ebene der Lehramtsausbildung und vielleicht auch für die Mathematikausbildung generell reflektiert werden. So sehr manches für die fachspezifische Modularität unserer Vorlesungen spricht, weil sie vordergründig unseren Ausbildungscurricula ein festes Gerüst verschaffen, so sind unbedingt auch Plattformen hinreichende Aufmerksamkeit zu widmen, auf denen die verschiedenen Module integrieren. Man mag einwenden, dass dies bei Diplomkandidaten spätestens bei der Erstellung der Arbeit geschieht. Doch dafür haben Lehramtskandidaten kaum noch Zeit, wenn mittlerweile schon in einzelnen Bundesländern eine nur schwer zu umgehende zeitliche Befristung (vier Monate) Verwaltungspraxis ist; und neuere Lehramtsausbildungsordnungen lassen nichts Gutes erahnen. Entsprechende Konsequenzen für die BachelorStudiengänge sind ebenfalls wohl kaum ausdiskutiert worden. Ohne die hohe Bedeutung der Strukturierung der Mathematik leugnen zu wollen, dieses Pri-

3 vgl. die Ausführungen Internationales und nationales Rahmenkonzept für die Erfassung von mathematischer Kompetenz in PISA des Konsortiums mit den Autoren Jürgen Baumert, Eckhard Klieme, Michael Neubrand, Manfred Prenzel, Ulrich Schiefele, Wolfgang Schneider, Klaus-Jürgen Tillmann und Manfred Weiß. Das Manuskript ist über das Max-Planck-Institut für Bildungsforschung in Berlin erhältlich. 
mat der Stoffstruktur darf die Anforderungen an die Lernstruktur nicht ignorieren. Hier ist eine geeignete Balance zu finden. Möglicherweise könnten diese problem- oder leitaspektorientierten Plattformen erste Begegnungen mit einer wissenschaftlichen Mathematik ermöglichen. Zu sehr ernüchternd sind Untersuchungen des Autors, was beispielsweise Lehramtskandidaten wirklich wissen. Wir dürfen davor die Augen nicht verschließen und müssen zu unserer Verantwortung stehen.

Weitere Informationen zu PISA können international über die Webside OECD-PISA ${ }^{4}$ bzw. über die Seite des Max-Planck-Instituts für Bildungsforschung ${ }^{5}$ abgerufen werden. Mathematikseitig sind in die $n a$ - tionale Expertengruppe (Mathematik) die folgenden Kollegen berufen worden: Prof. Dr. M. Neubrand (Flensburg), Prof. Dr. R. Biehler (Kassel), Prof. Dr. W. Blum (Kassel), Prof. Dr. E. CohorsFresenborg (Osnabrück), MR Dr. L. Flade (KuMi Sachsen-Anhalt), StD Dr. W. Löding (Hamburg), MR G. Möller (KuMi Düsseldorf) und Prof. Dr. A. Wynands (Bonn).

\section{Adresse des Autors}

Prof. Dr. Günter Törner

FB 11 - Mathematik

Universität Duisburg

47048 Duisburg

toerner@math.uni-duisburg.de
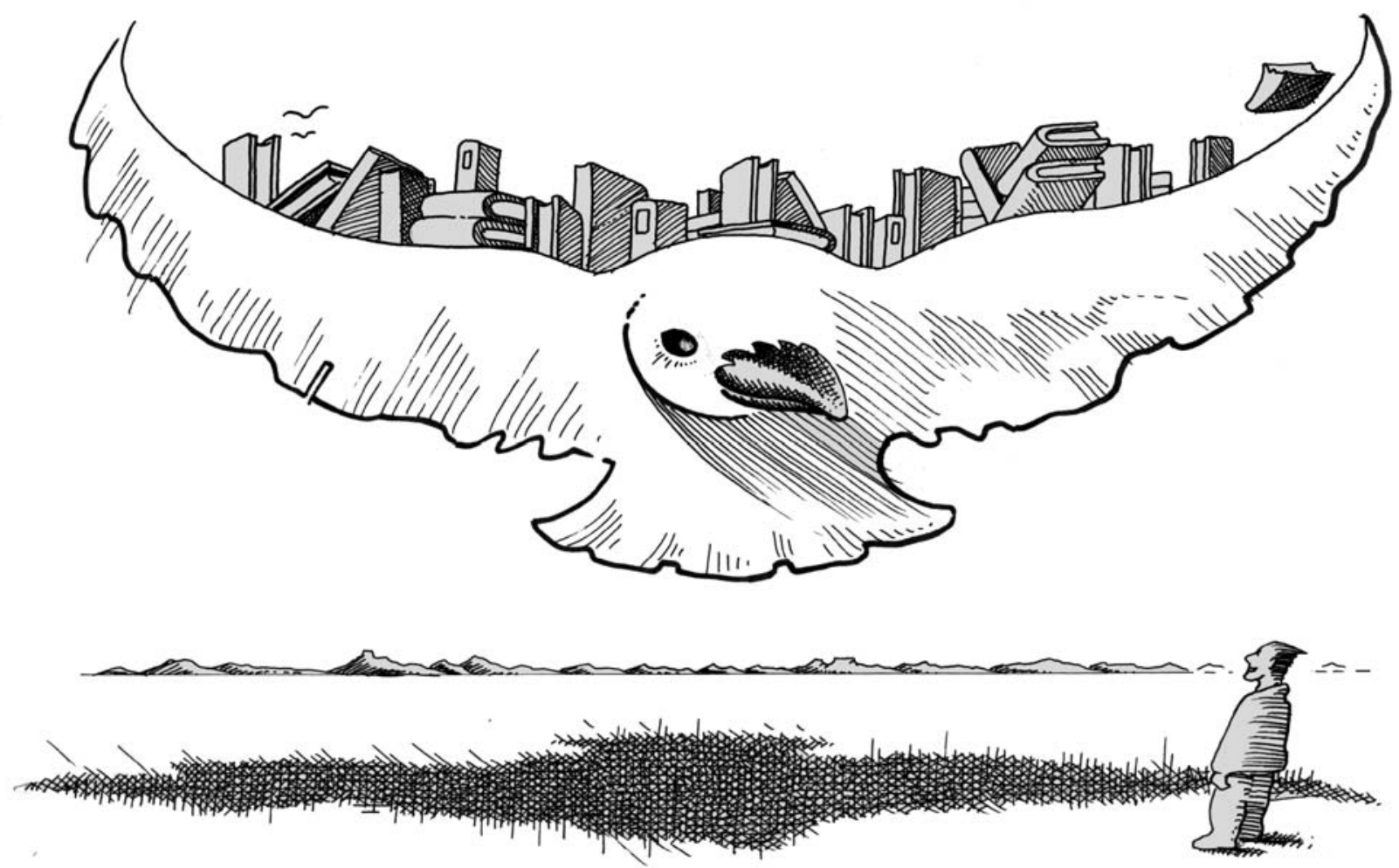

4 http://www.pisa.oecd.org

5 http://www.mpib-berlin.mpg.de/pisa 